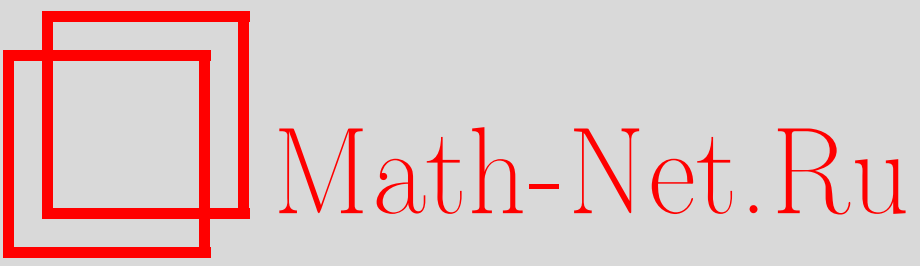

М. А. Васильев, О. В. Шейнкман, Конформная симметрия высших спинов для материальных полей в $(2+1)$-измерениях, ТM $\Phi, 2001$, том 128, номер 3, 378-394

DOI: https://doi.org/10.4213/tmf502

Использование Общероссийского математического портала Math-Net.Ru подразумевает, что вы прочитали и согласны с пользовательским соглашением

http://www. mathnet.ru/rus/agreement

Параметры загрузки:

IP : 54.80 .73 .141

26 апреля 2023 г., 15:12:48 
ТЕОРЕТИЧЕСКАЯ

И МАТЕМАТИЧЕСКАЯ

ФИЗИКА

Том 128, № 3

сентябрь, 2001

\author{
(C) 2001 г. М.А. Васильев*, О.В. Шейнкман*
}

\title{
КОНФОРМНАЯ СИММЕТРИЯ ВЫСШИХ СПИНОВ ДЛЯ МАТЕРИАЛЬНЫХ ПОЛЕЙ В $(2+1)$-ИЗМЕРЕНИЯХ
}

\begin{abstract}
В терминах вспомогательного фоковского модуля дана простая реализация конформной симметрии высших спинов на свободных безмассовых материальных полях в трех измерениях как для плоского, так и $A d S_{3}$-случая. Дуальность между неунитарными теоретико-полевыми представлениями конформной алгебры и унитарными представлениями синглетонного типа $3 d$-конформной алгебры $s p(4, \mathcal{R})$ явно сформулирована в терминах определенного преобразования Боголюбова.
\end{abstract}

\section{1. ВВЕДЕНИЕ}

AdS/CFT-соответствие [1], [2] предполагает дуальность между теориями гравитации в пространстве $A d S_{d}$ и $(d-1)$-мерными конформными теориями на границе $A d S_{d}$. Некоторое время назад была развита замкнутая теория взаимодействуюших безмассовых полей всех спинов в пространстве $A d S_{4}$ [3] (см. также [4], [5]). Так как калибровочная теория высших спинов содержит гравитацию и супергравитацию, можно предположить, что ей соответствует некоторая дуальная конформная теория, обладаюшая бесконечномерными $A d S_{4}$-симметриями высших спинов в качестве $3 d$-конформных симметрий высших спинов. В действительности $3 d$-конформная симметрия высших спинов была уже давно отож дествлена в [6] с $A d S_{4}$-алгеброй высших спинов [7], [8], тем самым предполагалось AdS/CFT-соответствие в теории высших спинов. В [6] был введен набор $3 d$-конформных калибровочных полей высших спинов, соответствующих конформной алгебре высших спинов. Однако $3 d$-калибровочные поля высших спинов не распространяются, т.е. их собственная динамика является топологической ${ }^{1)}$. Для того чтобы найти нетривиальную конформную теорию на гранище, необходимо реализовать $3 d$-конформную симметрию высших спинов на материальных полях. В этом и состоит цель настояшей статьи. Мы покажем, что конформная симметрия высших спинов допускает естественную реализацию на безмассовых скаляре и спиноре в трех измерениях.

\footnotetext{
1) Таким образом, термин "высшие спины" условный. Он означает, что калибровочные поля высших спинов и параметры симметрии являются тензорами высших рангов.
}

*Физический институт им. П.Н. Лебедева РАН, Москва, Россия. E-mail: vasiliev@lpi.ru, shayn@lpi.ru 
Эта реализация строится в терминах вспомогательного пространства Фока, оказывающегося дуальным синглетонному представлению в унитарном пространстве Фока [1] посредством определенного преобразования Боголюбова. Заметим, что предлагаемая реализация отличается от реализации, использованной для описания $3 d$-теории высших спинов в $[9],[10]$.

\section{3d-КОНФОРМНАЯ АЛГЕБРА ВЫСШИХ СПИНОВ}

Путем обобщения обычного соответствия между конформными симметриями в $d$ измерениях и $A d S$-симметриями в $d+1$ измерениях, $3 d$-конформные супералгебры высших спинов были отождествлены в [6] с $4 d A d S$-алгебрами высших спинов [7], [8]. Опишем конструкцию этого отождествления. Рассмотрим осцилляторы $\hat{a}_{\alpha}$ и $\hat{a}^{+\beta}$ с коммутационными соотношениями

$$
\left[\hat{a}_{\alpha}, \hat{a}^{+\beta}\right]=\delta_{\alpha}^{\beta}, \quad\left[\hat{a}_{\alpha}, \hat{a}_{\beta}\right]=\left[\hat{a}^{+\alpha}, \hat{a}^{+\beta}\right]=0,
$$

где $\alpha, \beta=1,2$. Изоморфизм $s o(3,2) \sim s p(4, \mathcal{R})$ позволяет использовать симплектическую реализацию $3 d$-конформных (и $A d S_{4}$ ) симметрий. Алгебра $s p(4, \mathcal{R})$ допускает стандартную осцилляторную реализацию в терминах билинейных комбинаций осцилляторов (2.1). Изоморфизм $s o(3,2) \sim s p(4, \mathcal{R})$ выражается соотношениями

$$
\begin{gathered}
\mathcal{L}_{n m}=\frac{1}{2} \epsilon_{n m k} \sigma^{k \alpha}{ }_{\beta} \mathcal{L}_{\alpha}{ }^{\beta}, \quad \mathcal{L}_{\alpha}{ }^{\beta}=\frac{1}{2}\left(\hat{a}_{\alpha} \hat{a}^{+\beta}+\hat{a}^{+\beta} \hat{a}_{\alpha}\right)-\frac{1}{4} \delta_{\alpha}^{\beta}\left(\hat{a}_{\gamma} \hat{a}^{+\gamma}+\hat{a}^{+\gamma} \hat{a}_{\gamma}\right), \\
\mathcal{P}_{n}=\sigma_{n}{ }^{\alpha \beta} \mathcal{P}_{\alpha \beta}, \quad \mathcal{P}_{\alpha \beta}=\frac{1}{2} \hat{a}_{\alpha} \hat{a}_{\beta}, \\
\mathcal{K}_{n}=\sigma_{n \alpha \beta} \mathcal{K}^{\alpha \beta}, \quad \mathcal{K}^{\alpha \beta}=\frac{1}{2} \hat{a}^{+\alpha} \hat{a}^{+\beta} \\
\mathcal{D}=\frac{1}{4}\left(\hat{a}_{\alpha} \hat{a}^{+\alpha}+\hat{a}^{+\alpha} \hat{a}_{\alpha}\right) .
\end{gathered}
$$

Здесь $n, m=0,1,2$ - индексы слоя, которые поднимаются и опускаются с помощью метрики Минковского $\eta_{n m}$ с сигнатурой $(+--)$, а $\sigma_{n}{ }^{\alpha \beta}=\sigma_{n}{ }^{\beta \alpha}=\left(I, \tau_{1}, \tau_{3}\right)^{\alpha \beta}$, где $\tau_{1}^{\alpha \beta}$, $\tau_{3}^{\alpha \beta}$-матрицы Паули. Спинорные индексы $\alpha, \beta$ поднимаются и опускаются с помощью $\epsilon_{\alpha \beta}=-\epsilon_{\beta \alpha}\left(\epsilon_{12}=\epsilon^{12}=1\right)$ как $c^{\alpha}=\epsilon^{\alpha \beta} c_{\beta}, c_{\beta}=\epsilon_{\alpha \beta} c^{\alpha}\left(\epsilon_{\alpha \beta}\right.$ - симплектическая форма $s p(2, \mathcal{R}) \sim s o(2,1))$. Матрицы $\sigma_{n}{ }^{\alpha \beta}$ удовлетворяют следуюшим соотношениям:

$$
\begin{gathered}
\sigma_{n}{ }^{\alpha \beta} \sigma_{m \alpha \beta}=2 \eta_{n m}, \quad \sigma_{n}{ }^{\alpha \beta} \sigma^{n} \alpha_{\alpha^{\prime} \beta^{\prime}}=\delta_{\alpha^{\prime}}^{\alpha} \delta_{\beta^{\prime}}^{\beta}+\alpha \leftrightarrow \beta, \\
\sigma_{n}{ }^{\alpha}{ }_{\beta} \sigma_{m}{ }^{\beta \gamma}=\eta_{n m} \epsilon^{\alpha \gamma}-\epsilon_{n m k} \sigma^{k \alpha \gamma} .
\end{gathered}
$$

Введенные в $(2.2)$ величины $\mathcal{L}_{\alpha}^{\beta}, \mathcal{P}_{\alpha \beta}, \mathcal{K}^{\alpha \beta}$ и $\mathcal{D}$ соответствуют генераторам лоренцевых поворотов, сдвигов Пуанкаре, специальных конформных преобразований и дилатации. $N=2$ суперсимметричное расширение $\operatorname{ssp}(2 \mid 4, \mathcal{R})$ алгебры $s p(4, \mathcal{R})$ допускает следуюшую полезную реализацию [11]. Расширим (2.1), добавив новый генератор $\hat{k}$, удовлетворяюший соотношениям

$$
\hat{k}^{2}=\hat{1}, \quad\left\{\hat{k}, \hat{a}_{\alpha}\right\}=\left\{\hat{k}, \hat{a}^{+\alpha}\right\}=0 .
$$


Супергенераторы $\mathcal{Q}_{j \alpha}, \mathcal{S}_{j}{ }^{\alpha}$, а также $u(1)$-заряд $\mathcal{J}$ имеют следуюший вид:

$$
\begin{aligned}
\mathcal{Q}_{j \alpha}=\frac{1}{\sqrt{2}}(i \hat{k})^{j} \hat{a}_{\alpha}, \quad \mathcal{S}_{j}{ }^{\alpha} & =\frac{1}{\sqrt{2}}(i \hat{k})^{j} \hat{a}^{+\alpha}, \quad j=0 \text { или } 1, \\
\mathcal{J} & =\frac{1}{4 i} \hat{k} .
\end{aligned}
$$

Обозначим (ассоциативную) обертьваюшую алгебру соотношений $(2.1),(2.4)$ через $A K_{2}$. Супералгебра Ли высших спинов $h g l(1 ; 1 \mid 4)$ определяется как то же самое линейное пространство $g l(1 ; 1 \mid 4)$, снабженное произведением, определяемым через (анти)коммутаторы в $A K_{2}:[\hat{f}, \hat{g}]_{ \pm}=\hat{f} \hat{g}-(-1)^{\pi(\hat{f}) \pi(\hat{g})} \hat{g} \hat{f}$. Каноническая $Z_{2}$-градуировка, выбранная в соответствии со стандартной взаимосвязью спина и статистики, определена по четности числа спинорных индексов:

$$
\hat{f}\left(-\hat{a}^{+},-\hat{a}, \hat{k}\right)=(-1)^{\pi(\hat{f})} \hat{f}\left(\hat{a}^{+}, \hat{a}, \hat{k}\right) .
$$

Левый модуль Фока алгебр $h g l(1 ; 1 \mid 4)$ и $A K_{2}$ может быть определен соотношениями

$$
\hat{a}_{\alpha}|0\rangle=0, \quad \hat{k}|0\rangle=|0\rangle .
$$

Базисными в нем являются векторы

$$
\hat{a}^{+\alpha_{1}} \ldots \hat{a}^{+\alpha_{l}}|0\rangle
$$

На практике, вместо того чтобы работать с операторной реализацией алгебры $A K_{2}$, удобно использовать ее формулировку в терминах звездочного произведения:

$$
\begin{aligned}
(f * g)\left(a^{+}, a\right)= & \frac{1}{\pi^{4}} \int d^{2} u d^{2} v d^{2} u^{+} d^{2} v^{+} e^{2 v_{\alpha} u^{+\alpha}-2 u_{\alpha} v^{+\alpha}} \times \\
& \times f\left(a^{+}+u^{+}, a+u\right) g\left(a^{+}+v^{+}, a+v\right),
\end{aligned}
$$

где $f\left(a^{+}, a\right)$ и $g\left(a^{+}, a\right)$ - функции (полиномы или формальные ряды) коммутируюших переменныхх $a^{+\alpha}$ и $\left.a_{\beta} .{ }^{2}\right)$

${ }^{2)}$ Интеграл определен таким образом, что

$$
\frac{1}{\pi^{2}} \int d^{2} v d^{2} u^{+} e^{2 v_{\alpha} u^{+\alpha}}=\frac{1}{\pi^{2}} \int d^{2} u d^{2} v^{+} e^{-2 u_{\alpha} v^{+\alpha}}=1
$$

и

$$
\begin{aligned}
& \int d^{2} v d^{2} u^{+} \frac{\partial}{\partial v_{\alpha}}\left(e^{2 v_{\alpha} u^{+\alpha}} f\left(v, u^{+}\right)\right)=\int d^{2} v d^{2} u^{+} \frac{\partial}{\partial u_{\alpha}^{+}}\left(e^{2 v_{\alpha} u^{+\alpha}} f\left(v, u^{+}\right)\right)= \\
& =\int d^{2} u d^{2} v^{+} \frac{\partial}{\partial u_{\alpha}}\left(e^{-2 u_{\alpha} v^{+\alpha}} g\left(u, v^{+}\right)\right)=\int d^{2} u d^{2} v^{+} \frac{\partial}{\partial v_{\alpha}^{+}}\left(e^{-2 u_{\alpha} v^{+\alpha}} g\left(u, v^{+}\right)\right)=0
\end{aligned}
$$

для любых полиномов $f\left(v, u^{+}\right)$и $g\left(u, v^{+}\right)$. Определение через осциллирующие экспоненты легко может быть получено с помощью соответствующей замены переменных. 
Формула (2.10) описывает ассоциативную алгебру с определяющими соотношениями

$$
\begin{gathered}
{\left[a_{\alpha}, a^{+\beta}\right]_{*}=\delta_{\alpha}^{\beta},} \\
{\left[a_{\alpha}, a_{\beta}\right]_{*}=\left[a^{+\alpha}, a^{+\beta}\right]_{*}=0 .}
\end{gathered}
$$

Определенное таким образом звездочное произведение описывает произведение симметризованных полиномов от осцилляторов в терминах символов операторов и реализует подалгебру $A_{2} \subset A K_{2}$, порожденную элементами, не зависящими от $\hat{k}$. Вся алгебра $A K_{2}$ определена на функциях вида $f\left(a^{+}, a, k\right)=f_{0}\left(a^{+}, a\right)+f_{1}\left(a^{+}, a\right) k$ с помощью формулы (2.10), дополненной соотношением

$$
k * f\left(a^{+}, a, k\right)=f\left(-a^{+},-a, k\right) * k=f\left(-a^{+},-a, k\right) k, \quad k * k=1 .
$$

Алгебра $A K_{2}$ допускает инволюцию ${ }^{3)} \mu$, определяемую соотношениями

$$
\mu\left(a_{\alpha}\right)=i a_{\alpha}, \quad \mu\left(a^{+\alpha}\right)=i a^{+\alpha}, \quad \mu(k)=k .
$$

Любая инволюция $\mu$ ассоциативной алгебры $A$ порождает сопряжение

$$
\sigma(f)=-(i)^{\pi(f)} \mu(f)
$$

супералгебры Ли $L_{A}$, построенной из $A$ с помощью (анти)коммутаторов. Алгебра $h u(1 ; 1 \mid 4)$ - это вешественная форма алгебры $h g l(1 ; 1 \mid 4)$, выделяемая сопряжением (2.14). Элементы алгебры $h u(1 ; 1 \mid 4)$

$$
f^{\mathcal{R}}\left(a^{+}, a, k\right)=f_{0}^{\mathcal{R}}\left(a^{+}, a\right)+f_{1}^{\mathcal{R}}\left(a^{+}, a\right) k
$$

удовлетворяют соотношениям

$$
\begin{aligned}
& f_{0}^{\mathcal{R}}\left(a^{+}, a\right)=-(i)^{\pi\left(f_{0}^{\mathcal{R}}\right)} \overline{f_{0}^{\mathcal{R}}\left(-i a^{+},-i a\right)}, \\
& f_{1}^{\mathcal{R}}\left(a^{+}, a\right)=-(i)^{\pi\left(f_{1}^{\mathcal{R}}\right)} \overline{f_{1}^{\mathcal{R}}\left(i a^{+}, i a\right)} .
\end{aligned}
$$

Здесь черта обозначает комплексное сопряжение коэффициентов степенных рядов. Заметим, что суперконформные генераторы $(2.2),(2.5)$ и (2.6) удовлетворяют условиям вещественности $\sigma(f)=f$ и, следовательно, принадлежат $h u(1 ; 1 \mid 4)$.

Фоковское представление алгебры $A K_{2}$ является ее левым модулем $F^{1}$, порожденным векторами вида

$$
f *|0\rangle\langle 0|,
$$

где $|0\rangle\langle 0|$ - проектор на вакуумное пространство. Хорошо известно (см., например, $[12],[13])$, что в алгебре звездочных произведений фоковские проекторы допускают

\footnotetext{
3) Напомним, что инволюция - это инволютивный полулинейный антиавтоморфизм, т.е. $\mu(f * g)=\mu(g) * \mu(f), \mu^{2}=1, \mu(\alpha f)=\bar{\alpha} \mu(f)$ ( $\bar{\alpha}$ комплексно-сопряжено с $\left.\alpha\right)$. Сопряжение - это инволютивный полулинейный автоморфизм, т.е. $\sigma(f * g)=\sigma(f) * \sigma(g), \sigma^{2}=1, \sigma(\alpha f)=\bar{\alpha} \sigma(f)$. Любое сопряжение $\sigma$ с помощью условия $\sigma(f)=f$ выделяет вещественную форму комплексной алгебры.
} 
экспоненциальную реализацию. Фоковский вакуум, удовлетворяющий соотношениям (2.8), имеет вид

$$
|0\rangle\langle 0|=2(1+k) e^{-2 a_{\alpha} a^{+\alpha}} .
$$

Действительно, легко видеть, что

$$
\begin{gathered}
a_{\alpha} *|0\rangle\langle 0|=| 0\rangle\langle 0| * a^{+\alpha}=0, \\
k *|0\rangle\langle 0|=| 0\rangle\langle 0|* k=| 0\rangle\langle 0|, \quad| 0\rangle\langle 0|*| 0\rangle\langle 0|=| 0\rangle\langle 0| .
\end{gathered}
$$

Правый модуль Фока $F^{\mathrm{r}}$ алгебры $A K_{2}$ порожден векторами $|0\rangle\langle 0| * f$. Важными свойствами фоковского вакуума $|0\rangle\langle 0|$ являются его лоренц-инвариантность:

$$
\mathcal{L}_{\alpha}{ }^{\beta} *|0\rangle\langle 0|=| 0\rangle\langle 0| * \mathcal{L}_{\alpha}^{\beta}=0,
$$

и наличие определенного конформного веса 1/2:

$$
\mathcal{D} *|0\rangle\langle 0|=| 0\rangle\left\langle 0\left|* \mathcal{D}=\frac{1}{2}\right| 0\right\rangle\langle 0| .
$$

Заметим, что $\mu$-сопряженный фоковский модуль $\mu(|0\rangle\langle 0|)=2(1+k) e^{2 a_{\alpha} a^{+\alpha}}$ отличается от $|0\rangle\langle 0|$. Более того, он принадлежит к другому сектору алгебры звездочных произведений, потому что $|0\rangle\langle 0| * \mu(|0\rangle\langle 0|)=\infty$. Это обстоятельство не является сушественным с точки зрения настояшей статьи, где рассматриваются только свободные поля (т.е. модули материальных полей не перемножаются), но должно приниматься во внимание при построении нелинейной теории, описывающей взаимодействия, которые могли бы обладать конфорными симметриями высших спинов.

\section{3. КОНФОРМНО-ИНВАРИАНТНЫЙ ВАКУУМ}

Рассмотрим 1-форму $\omega(x)$, принимаюшую значения в алгебре высших спинов $A K_{2}$, т.е. являюшуюся производяшей функцией калибровочных конформных полей высших спинов

$$
\omega(x)=\sum_{q=0,1} \sum_{l, r=0}^{\infty} \frac{1}{l ! r !} \omega_{q}(x)_{\alpha_{1} \ldots \alpha_{l},}{ }^{\beta_{1} \ldots \beta_{r}} a^{+\alpha_{1}} \ldots a^{+\alpha_{l}} a_{\beta_{1}} \ldots a_{\beta_{r}}(k)^{q}
$$

Уравнение нулевой кривизны

$$
d \omega=\omega \wedge * \omega
$$

инвариантно относительно конформных калибровочных преобразований высших спинов

$$
\delta \omega=d \epsilon-[\omega, \epsilon]_{*},
$$

где $\epsilon\left(a^{+}, a, k \mid x\right)$ - бесконечно малый параметр калибровочной симметрии и $d=$ $d x^{\underline{n}} \frac{\partial}{\partial x^{\underline{n}}}$ (подчеркнутые индексы $\underline{m}, \underline{n}=0,1,2$ используются для компонент дифиференциальных форм). Любое вакуумное решение $\omega_{0}$ уравнения (3.2) нарушает 
локальную симметрию высших спинов до подалгебры стабильности с бесконечно малым параметром $\epsilon_{0}\left(a^{+}, a, k \mid x\right)$, удовлетворяюшим уравнению

$$
d \epsilon_{0}-\left[\omega_{0}, \epsilon_{0}\right]_{*}=0
$$

Совместность этого уравнения обеспечивается уравнением (3.2).

Локально уравнение (3.2) допускает чисто калибровочное решение

$$
\omega_{0}=-g^{-1} * d g
$$

Здесь $g\left(a^{+}, a, k \mid x\right)$ - некоторый обратимый элемент алгебры $A K_{2}$, т.е. $g^{-1} * g=$ $g * g^{-1}=1$. Для $\omega_{0}$ в (3.5) обшее решение уравнения (3.4) имеет вид

$$
\epsilon_{0}(x)=g^{-1}(x) * \xi * g(x),
$$

где $\xi\left(a^{+}, a, k\right)=\xi_{0}\left(a^{+}, a\right)+\xi_{1}\left(a^{+}, a\right) k$ - произвольный, не зависящий от $x$ элемент, играюший роль начальных данных для уравнения (3.4):

$$
\left.\epsilon_{0}\left(a^{+}, a, k \mid x\right)\right|_{x=x_{0}}=\xi\left(a^{+}, a, k\right)
$$

для такой точки $x_{0}$, что $g\left(x_{0}\right)=1$. Таким образом, $h u(1 ; 1 \mid 4)$ действительно является алгеброй глобальной симметрии, оставляюшей инвариантным вакумное решение. Она содержит $N=2$ конформную глобальную супералгебру, порождаемую генераторами $(2.2),(2.5)$ и $(2.6)$.

Как обычно, гравитационные поля (т.е. триада и лоренцева связность) ассоциируются с генераторами сдвигов и лоренцевыми поворотами в пуанкаре- или $A d S$-подалгебре конформной алгебры. В плоском пространстве Минковского можно положить

$$
\omega_{0}=\omega_{f}=\frac{1}{2} d x^{\underline{n}} \sigma_{\underline{n}}^{\alpha \beta} a_{\alpha} a_{\beta}
$$

тем самым считая лоренцеву связность равной нулю, здесь $\sigma_{\underline{n}}{ }^{\alpha \beta}=\sigma_{n}{ }^{\alpha \beta}$. Функция $g_{f}$, порождаюшая плоское гравитационное поле (3.8), имеет вид

$$
g_{f}=e^{-\frac{x^{\alpha \beta}}{2} a_{\alpha} a_{\beta}}
$$

где мы использовали обозначения

$$
x^{\alpha \beta}=x^{\underline{n}} \sigma_{\underline{n}}{ }^{\alpha \beta}, \quad x^{\underline{n}}=\frac{1}{2} \sigma^{\underline{n}}{ }_{\alpha \beta} x^{\alpha \beta} .
$$

С помощью осцилляторной реализации $3 d$-конформной алгебры $(2.2)$ вложение алгебры $A d S_{3} o(2,2) \subset o(3,2)$ строится следующим образом:

$$
\begin{aligned}
& \mathcal{L}_{\alpha}^{\beta}=a_{\alpha} a^{+\beta}-\frac{1}{2} \delta_{\alpha}^{\beta} a_{\gamma} a^{+\gamma}, \\
& \mathcal{P}_{\alpha \beta}=\frac{1}{2}\left(a_{\alpha} a_{\beta}+\frac{\lambda^{2}}{4} a_{\alpha}^{+} a_{\beta}^{+}\right) .
\end{aligned}
$$


$A d S_{3}$-гравитационные поля отождествляются с 1-формами, принимаюшими значения в алгебре $A d S_{3}$,

$$
\begin{aligned}
\omega_{0}= & \omega_{A d S_{3}}=d x^{\underline{n}}\left(\frac{1}{2} e_{\underline{n}}^{\alpha \beta}(x)\left(a_{\alpha} a_{\beta}+\frac{\lambda^{2}}{4} a_{\alpha}^{+} a_{\beta}^{+}\right)+\right. \\
& \left.+\omega_{\underline{n}}^{\alpha}{ }_{\beta}(x)\left(a_{\alpha} a^{+\beta}-\frac{1}{2} \delta_{\alpha}^{\beta} a_{\gamma} a^{+\gamma}\right)\right)
\end{aligned}
$$

где $e_{\underline{n}}{ }^{\alpha \beta}(x)$ и $\omega_{\underline{n}}{ }^{\alpha}{ }_{\beta}(x)$ являются соответственно триадой и лоренцевой связностью пространства $A d S_{3}$. Конкретная форма гравитационных полей $A d S_{3}$, удовлетворяющих вакуумному уравнению (3.2) и соответствуюших "стереографическим" координатам пространства $A d S_{3}$, имеет вид

$$
e_{\underline{n}}^{\alpha \beta}=\frac{4}{\left(4+\lambda^{2} x^{2}\right)^{2}}\left(\left(4-\lambda^{2} x^{2}\right) \delta \underline{\underline{k}}+4 \lambda x \underline{\underline{m}} \epsilon_{\underline{n} \underline{m}}^{\underline{k}}+2 \lambda^{2} x_{\underline{n}} x^{\underline{k}}\right) \sigma_{\underline{k}}{ }^{\alpha \beta}
$$

и

$$
\omega_{\underline{n}}^{\alpha}{ }_{\beta}=-\frac{\lambda}{2} e_{\underline{n}}^{\alpha}{ }_{\beta},
$$

где

$$
x^{2}=\eta \frac{n m}{x_{\underline{n}}} x_{\underline{m}}=\frac{1}{2} x^{\alpha \beta} x_{\alpha \beta} .
$$

Метрический тензор $A d S_{3}$ записывается как

$$
g_{\underline{n m}}=\frac{1}{2} e_{\underline{n}}^{\alpha \beta} e_{\underline{m} \alpha \beta}=16 \frac{\eta_{\underline{n m}}}{\left(4+\lambda^{2} x^{2}\right)^{2}} .
$$

Следует отметить, что, хотя метрический тензор построен из $\omega_{0}$ и поэтому инвариантен относительно глобальных преобразований симметрии, эти симметрии необязательно связаны с векторами Киллинга метрического тензора. Это верно только для лоренцевых поворотов и (пуанкаре- или $A d S$-) сдвигов.

Легко видеть, что представление (3.5) для $A d S_{3}$-вакумных полей (3.12)-(3.14) дается калибровочной функцией

$$
g_{A d S_{3}}=\frac{1}{2} \sqrt{4+\lambda^{2} x^{2}} \exp \left(-\frac{x^{\alpha \beta}}{2}\left(a_{\alpha} a_{\beta}+\frac{\lambda^{2}}{4} a_{\alpha}^{+} a_{\beta}^{+}-\lambda a_{\alpha}^{+} a_{\beta}\right)\right)
$$

обладющей обратной к ней функцией

$$
g_{A d S_{3}}^{-1}=\frac{1}{2} \sqrt{4+\lambda^{2} x^{2}} \exp \left(\frac{x^{\alpha \beta}}{2}\left(a_{\alpha} a_{\beta}+\frac{\lambda^{2}}{4} a_{\alpha}^{+} a_{\beta}^{+}-\lambda a_{\alpha}^{+} a_{\beta}\right)\right)
$$

Заметим, что в плоском пределе $\lambda \rightarrow 0$ возникает плоская калибровочная функция (3.9). 


\section{3d-КОНФОРМНЫЕ ПОЛЕВЫЕ УРАВНЕНИЯ}

Как было показано ранее, уравнения движения безмассовых [14] и массивных [15] полей в пространстве $A d S_{3}$ допускают формулировку в терминах производяших функций

$$
C(y \mid x)=\sum_{l=0} \frac{1}{l !} C(x)_{\alpha_{1} \ldots \alpha_{l}} y^{\alpha_{1}} \ldots y^{\alpha_{l}}
$$

со вспомогательными спинорными переменными $y^{\alpha}$. Плоский предел свободных уравнений движения для скалярного и спинорного безмассовых полей [14] имеет вид

$$
d C(y \mid x)=\frac{1}{2} d x^{\underline{n}}{\sigma_{\underline{n}}}^{\alpha \beta} \frac{1}{\partial y^{\alpha} \partial y^{\beta}} C(y \mid x) .
$$

Это уравнение распадается на две независимые подсистемы: для четных функций $C_{\mathrm{e}}(-y \mid x)=C_{\mathrm{e}}(y \mid x)$ и нечетных функций $C_{\mathrm{o}}(-y \mid x)=-C_{\mathrm{o}}(y \mid x)$, которые описывают соответственно безмассовые скаляр и спинор.

В работе [9] спинорные переменные $y^{\alpha}$ интерпретировались как образуюшие $A d S_{3^{-}}$ алгебры высших спинов, в то время как 0-формы $C(y \mid x)$ принимали значения в так называемом твистованном присоединенном представлении этой алгебры. В работе [10] было показано, что соответствуюшая модификация формулировки [9] позволяет единым образом описывать как безмассовые, так и массивные материальные поля. Однако такая формулировка не обладает явными конформными симметриями, ожидаемыми в безмассовом случае. Ключевое наблюдение настоящей статьи состоит в том, что в безмассовом случае те же уравнения (4.2) допускают другую реализацию в фоковскомпространстве (2.16), в которой конформные симметрии высших спинов системы становятся явными.

Рассмотрим вектор в пространстве Фока

$$
\left|\Phi\left(a^{+} \mid x\right)\right\rangle=C\left(a^{+} \mid x\right) *|0\rangle\langle 0|, \quad C\left(a^{+} \mid x\right)=\sum_{l=0}^{\infty} \frac{1}{l !} c_{\alpha(l)}(x) \underbrace{a^{+\alpha} \ldots a^{+\alpha}}_{l},
$$

где 0-формы $c_{\alpha(l)}(x)$ являются полностью симметричными мультиспинорами ${ }^{4}$. Система уравнений

$$
d|\Phi\rangle-\omega *|\Phi\rangle=0
$$

компактно описывает уравнения Клейна-Гордона и Дирака для скалярного поля $c(x)$ и спинорного поля $c_{\alpha}(x)$ при условии, что выполнено уравнение $(3.2)$, гарантирующее формальную совместность (4.4). Действительно, выбор $\omega=\omega_{f}$ в форме (3.8) делает уравнение (4.4) эквивалентным уравнению (4.2). Заметим, что уравнения на компоненты полей $c_{\alpha(n)}(x)$ масштабно- и лоренш-инвариантны вследствие лоренш-инвариантности (2.19) и определенной масштабной размерности (2.20) вакуума $|0\rangle\langle 0|$.

\footnotetext{
4) Мы следуем соглашениям [16], удобным для покомпонентного анализа сложных тензорных структур: верхние и нижние индексы, обозначенные одной и той же буквой, должны быть сначала раздельно симметризованы, а затем их максимально возможное количество должно быть свернуто; число индексов может быть указано в скобках с использованием, например, обозначения $\alpha(l)$ вместо $l$-кратного повторения индекса $\alpha$.
}

3 Теоретическая и математическая физика, т. 128, № 3, 2001 г. 
Напомним, что, как показано в [14] для случая $A d S_{3}$, уравнения (4.2) приводят к динамическим уравнениям на безмассовые материальные поля, отождествляемые со спинорами ранга 0 и ранга 1 :

$$
\begin{gathered}
c(x)=C(0 \mid x) \\
c_{\alpha}(x)=\left.\frac{\partial}{\partial a^{+\alpha}} C\left(a^{+} \mid x\right)\right|_{a^{+\alpha}=0}
\end{gathered}
$$

и выражают спиноры всех высших рангов в (4.3) через высшие производные $c(x)$ и $c_{\alpha}(x)$. В простейшем случае плоского пространства это можно увидеть следующим образом. Подставляя $\omega_{f}$ в (4.4), находим

$$
\partial_{\underline{n}} c_{\alpha(l)}-\frac{1}{2} \sigma_{\underline{n}}^{\alpha \alpha} c_{\alpha(l+2)}=0
$$

Первые два уравнения для четных $l$, т.е. для $l=0$ и $l=2$, имеют вид

$$
\begin{aligned}
\partial_{\underline{n}} c-\frac{1}{2} \sigma_{\underline{n}}^{\alpha \alpha} c_{\alpha(2)} & =0, \\
\partial_{\underline{n}} c_{\alpha(2)}-\frac{1}{2} \sigma_{\underline{n}}{ }^{\alpha \alpha} c_{\alpha(4)} & =0 .
\end{aligned}
$$

Из (4.8) получаем, что $c_{\alpha(2)}=\sigma^{\underline{n}}{ }_{\alpha \alpha} \partial_{\underline{n}} c$. Подставляя это в (4.9) и умножая на $\sigma \underline{m}_{\beta \beta}$, имеем

$$
\sigma^{\underline{n}}{ }_{\alpha \alpha} \sigma^{\underline{m}}{ }_{\beta \beta} \partial_{\underline{n}} \partial_{\underline{m}} c=c_{\alpha(2) \beta(2)} \cdot
$$

Условие полной симметричности мультиспинора $c_{\alpha(2) \beta(2)}$ эквивалентно уравнению Клейна-Гордона на $c(x)$ :

$$
\square c(x)=0
$$

Уравнения для всех остальных четных значений $l$ не накладывают дальнейших дифференциальных уравнений на $c(x)$, а только выражают высшие мультиспиноры через высшие производные $c(x)$. Можно убедиться непосредственно, что окончательное выражение для четной части $C$, обозначенной $C_{\mathrm{e}}$, приобретает вид

$$
C_{\mathrm{e}}\left(a^{+} \mid x\right)=\sum_{q=0}^{\infty} \frac{1}{(2 q) !} \sigma^{\underline{n}_{1}} \alpha \alpha \ldots \sigma^{\underline{n}_{q}} \alpha \alpha \partial_{\underline{n}_{1}} \ldots \partial_{\underline{n}_{q}} c(x) \underbrace{a^{+\alpha} \ldots a^{+\alpha}}_{2 q} .
$$

Ситуация с фермионом аналогична. Начиная с уравнения (4.7) при $l=1$, получаем

$$
c_{\alpha \beta(2)}=\sigma^{\underline{n}}{ }_{\beta \beta} \partial_{\underline{n}} c_{\alpha} .
$$

Далее условие симметричности мультиспинора третьего ранга $c_{\alpha \beta(2)}$ накладывает уравнение Дирака на $c_{\alpha}(x)$ :

$$
\sigma^{\underline{n} \beta}{ }_{\alpha} \partial_{\underline{n}} c_{\beta}(x)=0 .
$$


Все остальные уравнения для нечетных значений $l$ выражают высшие спиноры через высшие производные $c_{\alpha}(x)$. Для нечетной части $C$, обозначенной $C_{\mathrm{o}}$, получаем

$$
C_{\mathrm{o}}\left(a^{+} \mid x\right)=\sum_{q=0}^{\infty} \frac{1}{(2 q+1) !} \sigma^{\underline{n}_{1}} \alpha \alpha \ldots \sigma^{\underline{\underline{n}}} \alpha \alpha \partial_{\underline{n}_{1}} \ldots \partial_{\underline{n}_{q}} c_{\alpha}(x) \underbrace{a^{+\alpha} \ldots a^{+\alpha}}_{2 q+1} .
$$

Таким образом, мы заключаем, что для плоской связности $\omega_{f}$ система (4.4) эквивалентна

$$
\begin{aligned}
\left|\Phi\left(a^{+} \mid x\right)\right\rangle= & \sum_{q=0}^{\infty} \frac{1}{(2 q) !} \sigma^{\underline{n}_{1}} \alpha \alpha \ldots \sigma^{\underline{n}} \alpha \times \\
& \times \partial_{\underline{n}_{1}} \ldots \partial_{\underline{n}_{q}}\left(c(x)+\frac{1}{2 q+1} c_{\alpha}(x) a^{+\alpha}\right) \underbrace{a^{+\alpha} \ldots a^{+\alpha}}_{2 q} *|0\rangle\langle 0|
\end{aligned}
$$

вместе с динамическими уравнениями (4.11) и (4.14).

Аналогично для $\omega_{A d S_{3}}$ система (4.4) эквивалентна безмассовым уравнениям Клейна-Гордона и Дирака в пространстве $A d S_{3}$ :

$$
\begin{gathered}
\left(g^{\underline{n m}} D_{\underline{n}} D_{\underline{m}}-\frac{3}{4} \lambda^{2}\right) c(x)=0, \\
e^{\underline{n} \beta}{ }_{\alpha} D_{\underline{n}} c_{\beta}(x)=0
\end{gathered}
$$

вместе с уравнением

$$
\begin{aligned}
\left|\Phi\left(a^{+} \mid x\right)\right\rangle= & \sum_{q=0}^{\infty} \frac{1}{(2 q) !} e^{\underline{n}_{1}} \alpha \alpha \ldots e^{\underline{n}_{q}} \alpha \alpha \times \\
& \times D_{\underline{n}_{1}} \ldots D_{\underline{n}_{q}}\left(c(x)+\frac{1}{2 q+1} c_{\alpha}(x) a^{+\alpha}\right) \underbrace{a^{+\alpha} \ldots a^{+\alpha}}_{2 q} *|0\rangle\langle 0|,
\end{aligned}
$$

где $D_{\underline{n}}$ обозначает лоренц-ковариантную производную в пространстве $A d S_{3}$ :

$$
\begin{gathered}
D_{\underline{n}} c_{\alpha(l)}=\partial_{\underline{n}} c_{\alpha(l)}+l \omega_{\underline{n}}^{\beta}{ }_{\alpha} c_{\alpha(l-1) \beta}, \\
D_{\underline{n}} e_{\underline{m}}^{\alpha \alpha}-\underline{n} \leftrightarrow \underline{m}=0 .
\end{gathered}
$$

Заметим, что, как обычно, мы отождествляем безмассовые скаляр и спинор в пространстве $A d S_{3}$ с конформными полями. В соответствии с этой договоренностью безмассовое скалярное поле обладает ненулевым массоподобным членом $m_{0}^{2}=-\left(\lambda^{2} / 4\right) d(d-2)$, что находится в согласии с $(4.17)$ для $d=3$. 


\section{3d-КОНФОРМНЫЕ СИММЕТРИИ ВЫСШИХ СПИНОВ}

Система уравнений (3.2) и (4.4) инвариантна относительно бесконечномерных локальных конформных симметрий высших спинов вида (3.3) и

$$
\delta|\Phi\rangle=\epsilon *|\Phi\rangle
$$

(здесь $\left.\epsilon=\epsilon\left(a^{+}, a, k \mid x\right)\right)$. При фиксации конкретного вакуумного решения $\omega=\omega_{0}$ локальная симметрия высших спинов (5.1) нарушается до глобальной симметрии высших спинов (3.6). Поэтому система (4.7) и ее $A d S_{3}$-аналог инвариантны относительно бесконечномерной алгебры глобальных $3 d$-конформных симметрий

$$
\delta|\Phi\rangle=\epsilon_{0} *|\Phi\rangle
$$

высших спинов $h u(1 ; 1 \mid 4)$, где $\epsilon_{0}$ удовлетворяет уравнению (3.4) с плоской связностью (3.8) или $A d S_{3}$-связностью (3.12). Когда старшие компоненты $c_{\alpha(l)}$ выражены через высшие производные динамических полей спина 0 и $1 / 2$ с помощью уравнений (4.16) и (4.19), это гарантирует инвариантность безмассовых уравнений Клейна-Гордона и Дирака (4.11), (4.14) и (4.17), (4.18) относительно конформных симметрий высших спинов. Таким образом, переформулировка безмассовых уравнений Клейна-Гордона и Дирака в форме условий ковариантного постоянства (4.4), дополненных уравнениями нулевой кривизны (3.2), делает явными конформные симметрии высших спинов.

$N=2$ суперсимметричная конформная алгебра $\operatorname{osp}(2 \mid 4, \mathcal{R})$, порождаемая элементами $(2.2),(2.5)$ и $(2.6)$, является конечномерной подалгеброй алгебры $3 d$-конформных симметрий высших спинов $h u(1 ; 1 \mid 4)$. Вместе с $u(1)$-фактором, генерируемым единичным элементом в алгебре $h u(1 ; 1 \mid 4)=u(1) \oplus h s u(1 ; 1 \mid 4)$, алгебра $u(1) \oplus \operatorname{osp}(2 \mid$ $4, \mathcal{R})$ является максимальной конечномерной подалгеброй $h u(1 ; 1 \mid 4)$. Из формул $(4.16)$ или (4.19), а также из квантово-механической нелокальности звездочного произведения $(2.10)$ следует, что чем выше степень $\epsilon_{0}\left(a^{+}, a, k \mid x\right)$ как полинома по $a_{\alpha}$ и $a^{+\beta}$, тем более высокие степени пространственно-временных производных появляются в выражениях для конформных преобразований высших спинов. Это служит конкретной иллюстрацией того хорошо известного факта, что симметрии высших спинов вовлекают высшие производные динамических полей.

Явная форма преобразований может быть получена подстановкой $(4.16)((4.19)$ в случае $\left.A d S_{3}\right)$ в (5.2). На практике оказьвается наиболее удобным вычислить конформные преобразования высших спинов для производяшего параметра

$$
\xi^{j}\left(a^{+}, a, k ; h^{+}, h\right)=\xi e^{a^{+\alpha} h_{\alpha}+a_{\alpha} h^{+\alpha}}(k)^{j},
$$

где $\xi$-бесконечно малый параметр, а $h_{\alpha}, h^{+\alpha}$ - спинорные "источники". Полиномиальные параметры симметрии могут быть получены дифференцированием $\xi^{j}\left(a^{+}, a, k ; h^{+}, h\right)$ по $h_{\alpha}$ и $h^{+\alpha}$. Используя (3.6), (3.17) и формулу для звездочного произведения $(2.10)$, после вычисления элементарных гауссовых интегралов получаем

$$
\begin{gathered}
\epsilon_{0}\left(a^{+}, a, k ; h^{+}, h \mid x\right)=\xi \exp \left(\frac { 1 } { 8 + 2 \lambda ^ { 2 } x ^ { 2 } } \left(x ^ { \alpha \alpha } \left(8 a_{\alpha} h_{\alpha}+4 \lambda a_{\alpha} h_{\alpha}^{+}-4 \lambda a_{\alpha}^{+} h_{\alpha}-\right.\right.\right. \\
\left.\left.\left.-2 \lambda^{2} a_{\alpha}^{+} h_{\alpha}^{+}\right)+8 a^{+\alpha} h_{\alpha}+8 a_{\alpha} h^{+\alpha}+4 \lambda x^{2} a^{\alpha} h_{\alpha}+\lambda^{3} x^{2} a_{\alpha}^{+} h^{+\alpha}\right)\right)(k)^{j} .
\end{gathered}
$$


Подстановка $\epsilon_{0}$ в $(5.2)$ позволяет записать глобальные конформные преобразования высших спинов, индуцированные параметром $\xi^{j}\left(a^{+}, a, k ; h^{+}, h\right)(5.3)$ :

$$
\begin{gathered}
\delta\left|\Phi\left(a^{+} \mid x\right)\right\rangle=\xi \exp \left(\frac { 1 } { 8 + 2 \lambda ^ { 2 } x ^ { 2 } } \left(x^{\alpha \alpha}\left(4 h_{\alpha} h_{\alpha}-\lambda^{2} h_{\alpha}^{+} h_{\alpha}^{+}-4 \lambda h_{\alpha} a_{\alpha}^{+}-2 \lambda^{2} h_{\alpha}^{+} a_{\alpha}^{+}\right)+\right.\right. \\
\left.\left.+8 a^{+\alpha} h_{\alpha}+\lambda^{3} x^{2} a_{\alpha}^{+} h^{+\alpha}+\left(4-\lambda^{2} x^{2}\right) h^{+\alpha} h_{\alpha}\right)\right) \times \\
\times C\left((-1)^{j}\left(a^{+\alpha}+\frac{8 x^{\alpha \beta} h_{\beta}+4 \lambda x^{\alpha \beta} h_{\beta}^{+}+8 h^{+\alpha}-4 \lambda x^{2} h^{\alpha}}{8+2 \lambda^{2} x^{2}}\right) \mid x\right) *|0\rangle\langle 0| .
\end{gathered}
$$

Полагая $\lambda=0$, находим формулу преобразования для плоского пространства:

$$
\begin{aligned}
\delta\left|\Phi\left(a^{+} \mid x\right)\right\rangle= & \xi \exp \left(\frac{1}{2} x^{\alpha \alpha} h_{\alpha} h_{\alpha}+a^{+\alpha} h_{\alpha}+\frac{1}{2} h^{+\alpha} h_{\alpha}\right) \times \\
& \times C\left((-1)^{j}\left(a^{+\alpha}+x^{\alpha \beta} h_{\beta}+h^{+\alpha}\right) \mid x\right) *|0\rangle\langle 0| .
\end{aligned}
$$

Дифференцируя по источникам $h_{\alpha}$ и $h^{+\alpha}$, получаем явные выражения для тех или иных глобальных конформных преобразований высших спинов. Для преобразований динамических полей (4.5), (4.6) это явное выражение упрошается еше больше. Например, бозонные преобразования динамического скаляра в плоском пространстве имеют вид

$$
\delta c=\xi e^{\frac{1}{2} x^{\alpha \alpha} h_{\alpha} h_{\alpha}+\frac{1}{2} h^{+\alpha} h_{\alpha}} C\left(x^{\alpha \beta} h_{\beta}+h^{+\alpha} \mid x\right),
$$

где в правую часть подставлено (4.12). Подчеркнем, что столь компактная форма конформных преобразований высших спинов является результатом переформулировки динамических уравнений в развернутой форме условий ковариантного постоянства, т.е. в терминах плоских сечений фоковского расслоения. Заметим, что попытка поиска конформных преобразований высших спинов “в лоб” быстро наталкивается на значительные трудности (особенно в $A d S_{3}$-случае).

Для не более чем квадратичных генераторов конформной супералгебры $(2.2),(2.5)$, (2.6) немедленно получаем для случая плоского пространства

$$
\begin{aligned}
\mathcal{L}_{\alpha}{ }^{\beta}\left|\Phi\left(a^{+} \mid x\right)\right\rangle= & \left(x^{\beta \gamma} \frac{\partial^{2}}{\partial a^{+\alpha} \partial a^{+\gamma}}+a^{+\beta} \frac{\partial}{\partial a^{+\alpha}}-\right. \\
& \left.-\frac{1}{2} \delta_{\alpha}^{\beta}\left(x^{\gamma \gamma} \frac{\partial^{2}}{\partial a^{+\gamma} \partial a^{+\gamma}}+a^{+\gamma} \frac{\partial}{\partial a^{+\gamma}}\right)\right)\left|\Phi\left(a^{+} \mid x\right)\right\rangle, \\
\mathcal{P}_{\alpha \alpha}\left|\Phi\left(a^{+} \mid x\right)\right\rangle= & \frac{1}{2} \frac{\partial^{2}}{\partial a^{+\alpha} \partial a^{+\alpha}}\left|\Phi\left(a^{+} \mid x\right)\right\rangle, \\
\mathcal{K}^{\alpha \alpha}\left|\Phi\left(a^{+} \mid x\right)\right\rangle= & \frac{1}{2}\left(x^{\alpha \alpha}+a^{+\alpha} a^{+\alpha}+\right. \\
& \left.+2 x^{\alpha \beta} a^{+\alpha} \frac{\partial}{\partial a^{+\beta}}+x^{\alpha \beta} x^{\alpha \beta} \frac{\partial^{2}}{\partial a^{+\beta} \partial a^{+\beta}}\right)\left|\Phi\left(a^{+} \mid x\right)\right\rangle, \\
\mathcal{D}\left|\Phi\left(a^{+} \mid x\right)\right\rangle= & \frac{1}{2}\left(1+a^{+\alpha} \frac{\partial}{\partial a^{+\alpha}}+x^{\alpha \alpha} \frac{\partial^{2}}{\partial a^{+\alpha} \partial a^{+\alpha}}\right)\left|\Phi\left(a^{+} \mid x\right)\right\rangle,
\end{aligned}
$$




$$
\begin{aligned}
\mathcal{Q}_{j \alpha}\left|\Phi\left(a^{+} \mid x\right)\right\rangle & =\frac{(i)^{j}}{\sqrt{2}} \frac{\partial}{\partial a^{+\alpha}}\left|\Phi\left((-1)^{j} a^{+} \mid x\right)\right\rangle, \\
\mathcal{S}_{j}{ }^{\alpha}\left|\Phi\left(a^{+} \mid x\right)\right\rangle & =\frac{(i)^{j}}{\sqrt{2}}\left(a^{+\alpha}+x^{\alpha \beta} \frac{\partial}{\partial a^{+\beta}}\right)\left|\Phi\left((-1)^{j} a^{+} \mid x\right)\right\rangle, \\
\mathcal{J}\left|\Phi\left(a^{+} \mid x\right)\right\rangle & =\frac{1}{4 i}\left|\Phi\left(-a^{+} \mid x\right)\right\rangle .
\end{aligned}
$$

Учитывая, что динамические уравнения на $\left|\Phi\left(a^{+} \mid x\right)\right\rangle$ имеют вид (4.2), можно заменить все вторые производные по $a^{+\alpha}$ на пространственно-временные производные. Это приводит к следующим стандартным выражениям:

$$
\begin{aligned}
\mathcal{L}_{n m}\left|\Phi\left(a^{+} \mid x\right)\right\rangle= & \left(x_{m} \partial_{n}-x_{n} \partial_{m}+\frac{1}{2} \epsilon_{n m k} \sigma_{\beta}^{k \alpha} a^{+\beta} \frac{\partial}{\partial a^{+\alpha}}\right)\left|\Phi\left(a^{+} \mid x\right)\right\rangle, \\
\mathcal{P}_{n}\left|\Phi\left(a^{+} \mid x\right)\right\rangle= & \partial_{n}\left|\Phi\left(a^{+} \mid x\right)\right\rangle \\
\mathcal{K}_{n}\left|\Phi\left(a^{+} \mid x\right)\right\rangle= & \left(x_{n}+2 x_{n} x^{k} \partial_{k}-x^{2} \partial_{n}+\right. \\
& \left.+\frac{1}{2} \sigma_{n \alpha \alpha}\left(a^{+\alpha} a^{+\alpha}+2 x^{\alpha \beta} a^{+\alpha} \frac{\partial}{\partial a^{+\beta}}\right)\right)\left|\Phi\left(a^{+} \mid x\right)\right\rangle \\
\mathcal{D}\left|\Phi\left(a^{+} \mid x\right)\right\rangle= & \left(x^{k} \partial_{k}+\frac{1}{2}+\frac{1}{2} a^{+\alpha} \frac{\partial}{\partial a^{+\alpha}}\right)\left|\Phi\left(a^{+} \mid x\right)\right\rangle .
\end{aligned}
$$

Для динамических скалярного и спинорного полей (4.5) и (4.6) плучаем ожидаемые результаты

$$
\begin{aligned}
\mathcal{L}_{n m} c & =\left(x_{m} \partial_{n}-x_{n} \partial_{m}\right) c \\
\mathcal{P}_{n} c & =\partial_{n} c \\
\mathcal{K}_{n} c & =\left(x_{n}+2 x_{n} x^{k} \partial_{k}-x^{2} \partial_{n}\right) c \\
\mathcal{D} c & =\left(\frac{1}{2}+x^{k} \partial_{k}\right) c \\
\mathcal{Q}_{j \alpha} c & =\frac{(-i)^{j}}{\sqrt{2}} c_{\alpha}, \\
\mathcal{S}_{j}^{\alpha} c & =\frac{(-i)^{j}}{\sqrt{2}} x^{\alpha \beta} c_{\beta}, \\
\mathcal{J} c & =-\frac{i}{4} c, \\
\mathcal{L}_{n m} c_{\alpha} & =\left(\left(x_{m} \partial_{n}-x_{n} \partial_{m}\right) \delta_{\alpha}^{\beta}+\frac{1}{2} \epsilon_{n m k} \sigma^{k \beta}\right) c_{\beta}, \\
\mathcal{P}_{n} c_{\alpha} & =\partial_{n} c_{\alpha}, \\
\mathcal{K}_{n} c_{\alpha} & =\left(\left(2 x_{n}+2 x_{n} x^{k} \partial_{k}-x^{2} \partial_{n}\right) \delta_{\alpha}^{\beta}-\epsilon_{n m k} x^{m} \sigma^{k \beta}{ }_{\alpha}\right) c_{\beta}, \\
\mathcal{D}_{\alpha} & =\left(1+x^{k} \partial_{k}\right) c_{\alpha}, \\
\mathcal{Q}_{j \alpha} c_{\beta} & =\frac{(i)^{j}}{\sqrt{2}} \sigma^{n}{ }_{\alpha \beta} \partial_{n} c,
\end{aligned}
$$




$$
\begin{aligned}
\mathcal{S}_{j}^{\alpha} c_{\beta} & =\frac{(i)^{j}}{\sqrt{2}}\left(\left(1+x^{k} \partial_{k}\right) \delta_{\beta}^{\alpha}+x_{n} \partial_{m} \epsilon^{n m k} \sigma_{k}{ }^{\alpha}{ }_{\beta}\right) c, \\
\mathcal{J} c_{\alpha} & =\frac{i}{4} c_{\alpha} .
\end{aligned}
$$

Заметим, что конкретная форма зависимости от пространственно-временных координат $x^{\alpha \beta}$ диктуется выбором калибровочной функции (3.9). Используемый подход применим для любой другой координатной системы и конформно-плоского фона (например, $\left.A d S_{3}\right)$.

\section{6. ДУАЛЬНОСТЬ СИНГЛЕТОННОЙ И ТЕОРЕТИКО-ПОЛЕВОЙ КАРТИН КАК ПРЕОБРАЗОВАНИЕ БОГОЛЮБОВА}

Формулировка релятивистской динамики высших спинов, предложенная в данной статье, использует модуль Фока $F(2.8)$, определенньй по отношению к вспомогательным осцилляторам, связанным с $3 d$-конформной супералгеброй $\operatorname{sp} p(2 \mid 4, \mathcal{R})$ соотношениями $(2.2),(2.5)$ и (2.6). Этот модуль Фока аналогичен фоковским представлениям алгебры $s p(4, \mathcal{R})$, совпадаюшим с синглетонами Дирака [1]. Отличие состоит в том, что синглетонное представление $S$ унитарно, а фоковский модуль $F-$ нет. В этом разделе мы посредством определенного преобразования Боголюбова покажем, что наш подход делает явной дуальность между этими двумя типами представлений. Эта параллель распространяется весьма далеко. В частности, теорема Флато-Фронсдала [17] о том, что тензорное произведение двух синглетонных представлений эквивалентно прямой сумме всех безмассовых $A d S_{4}$-представлений, приобретает простую динамическую интерпретацию в рамках развернутой формулировки динамики высших спинов.

Тот факт, что инволюция $\mu$ (2.13) отображает осцилляторы $a_{\alpha}$ и $a^{+\alpha}$ в самих себя, означает, что модуль Фока (4.3) не унитарен. Это соответствует тому, что вакуум $|0\rangle\langle 0|$ лоренш-инвариантен (2.19). Поэтому модуль (4.3) распадается в бесконечную сумму конечномерных представлений некомпактной лоренцевой $3 d$-алгебры $o(2,1)$, связанных с полями $c_{\alpha(l)}(x)$. (Напомним, что некомпактные алгебры не имеют конечномерных унитарных представлений.)

Унитарный модуль Фока алгебры $s p(4, \mathcal{R})$ строится из осцилляторов

$$
\left[\hat{b}_{i}^{ \pm}, \hat{b}_{j}^{ \pm}\right]=0, \quad\left[\hat{b}_{i}^{-}, \hat{b}_{j}^{+}\right]=\delta_{i j}, \quad i, j=1,2,
$$

удовлетворяюших условиям эрмитова сопряжения

$$
\left(\hat{b}_{i}^{ \pm}\right)^{\dagger}=\hat{b}_{i}^{\mp} .
$$

Соответствуюший фоковский вакуум $\left|0_{u}\right\rangle\left\langle 0_{u}\right|$ определяется как

$$
\hat{b}_{i}^{-}\left|0_{u}\right\rangle\left\langle 0_{u}|=0, \quad| 0_{u}\right\rangle\left\langle 0_{u}\right| \hat{b}_{i}^{+}=0 .
$$

Унитарные левый и правый модули Фока, построенные с помошью вакуума $\left|0_{u}\right\rangle\left\langle 0_{u}\right|$, идентифицируются соответственно с суперсинглетоном $S$ и сопряженным ему $\bar{S}$. (Суперсинглетон $S$ раскладывается на два неприводимых представления алгебры $s p(4, \mathcal{R})$, 
называемых "Рак" и "Ди" и связанных соответственно с подпространствами, построенными из $\left|0_{u}\right\rangle\left\langle 0_{u}\right|$ с помошю юетного и нечетного чисел операторов рождения [17].)

Два набора осцилляторов связаны следуюшим образом:

$$
\begin{gathered}
\hat{b}_{j}^{ \pm}=\frac{1}{\sqrt{2}}\left(\hat{a}_{j} \pm \hat{a}^{+j}\right), \\
\hat{a}_{j}=\frac{1}{\sqrt{2}}\left(\hat{b}_{j}^{+}+\hat{b}_{j}^{-}\right), \quad \hat{a}^{+j}=\frac{1}{\sqrt{2}}\left(\hat{b}_{j}^{+}-\hat{b}_{j}^{-}\right) .
\end{gathered}
$$

В терминах алгебры звездочного произведения (2.10) унитарный фоковский вакуум имеет вид

$$
\left|0_{u}\right\rangle\left\langle 0_{u}\right|=2(1+k) e^{-\delta^{i j}\left(a_{i} a_{j}-a_{i}^{+} a_{j}^{+}\right)} .
$$

Поэтому мы заключаем, что в нашем подходе имеется естественная дуальность между теоретико-полевым модулем $F$, используемым в развернутой формулировке $3 d$-конформной динамики, и унитарным модулем $S$.

Идея о наличии некоторой дуальности такого сорта развивалась ранее [18] для $4 d$-случая и является весьма интересной в контексте AdS/CFT-соответствия. Новым результатом является то, что в рамках предложенной формулировки $3 d$-конформной динамики эта дуальность принимает простую форму преобразований Боголюбова (6.4), (6.5). Следует подчеркнуть, что зависимость элементов поля $|\Phi(x)\rangle$ от пространственно-временных координат полностью определяется уравнением (4.4) в терминах значения этого поля в любой фиксированной точке $x_{0}$. Это значит, что модуль $\left|\Phi\left(x_{0}\right)\right\rangle$ содержит всю информацию о динамике $3 d$-конформных полей на массовой оболочке аналогично тому, как синглетонный модуль содержит всю информацию о квантовых состояниях свободной теории (на массовой оболочке). Мы полагаем, что этот феномен является весьма обшим и приведенная формулировка динамических систем в форме некоторых условий плоскостности (т.е. условий ковариантного постоянства или нулевой кривизны) обеспечит явную дуальность между классическим и квантовым описаниями динамических систем в обшем случае.

Дуальность между унитарной и развернутой формулировками динамических систем, обладаюших симметриями высших спинов, допускает интересное расширение на $A d S_{4}$-калибровочные теории высших спинов. Как было показано Флато и Фронсдалом [17], тензорное произведение синглетонных представлений равно прямой сумме унитарных представлений $s p(4, \mathcal{R})$, ассоциируемых с безмассовыми полями всех спинов в пространстве $A d S_{4}$. В частности, $A d S_{4}$-безмассовые поля всех спинов $s=0,1 / 2,1,3 / 2, \ldots$ появляются дважды в $S \otimes \bar{S}$. (Прямой анализ приведен также в статье [19].) Замечательно, что это в точности совпадает со спектром простейшей (т.е. без неабелевых калибровочных симметрий Янга-Миллса) суперсимметричной $A d S_{4}$-теории высших спинов, основанной на алгебре $h u(1 ; 1 \mid 4)$, которая была построена в работах [3], [20]. В работе [19] было показано, что этот факт означает, что алгебра $h u(1 ; 1 \mid 4)$ допускает унитарное представление, отвечаюшее одночастичным состояниям квантовой калибровочной теории высших спинов. В работе [21] было затем показано, что все самосогласованные калибровочные $4 d$-теории высших спинов 
с различными группами Янга-Миллса допускают представления, ассоциируемые с определенными тензорными произведениями пар синглетонов.

Теперь рассмотрим теоретико-полевую картину. Базис в тензорном произведении $F^{\mathrm{l}} \otimes F^{\mathrm{r}}$ левого и правого модулей Фока задается элементами вида

$$
a_{\alpha_{1}}^{+} * \cdots * a_{\alpha_{n}}^{+} *|0\rangle\langle 0| * a_{\beta_{1}} * \cdots * a_{\beta_{m}} .
$$

Тензорное произведение $F^{\mathrm{l}} \otimes F^{\mathrm{r}}$ можно отождествить с алгеброй эндоморфизмов пространства $F$. Алгебра звездочного произведения (2.10) также может быть интерпретирована как алгебра линейных операторов в $F$ и поэтому может быть отождествлена с $F^{1} \otimes F^{\mathrm{r}}$ ) . Важно, что $A d S_{4}$-калибровочные поля высших спинов - это калибровочные поля (1-формы), принимающие значения в этой алгебре. Тем самым возникает интересная динамическая реализация теоремы Флато-Фронсдала. Более того, в недавней работе [22] было показано, что, в согласии с AdC/CFT-соответствием [2], набор $A d S_{4}$-калибровочных полей высших спинов находится во взаимно однозначном соответствии с набором $3 d$-конформных токов высших спинов.

Важный вопрос о явной форме AdS/CFT-соответствия в рамках калибровочной теории высших спинов требует более тшательного анализа динамики высших спинов и будет рассмотрен позже. Одна из связанных с этим соответствием проблем состоит в том, что предложенная в данной работе фоковская реализация $3 d-$-уравнений отличается от подхода к нелинейной $3 d$-теории высших спинов, предложенного в [9], [10], где уравнение (4.4) было сформулировано в терминах твистованного присоединенного представления $A d S_{3}$-алгебры высших спинов, реализованного в соответствии с (4.1) меньшим набором осцилляторов $y^{\alpha}$. Так как формулировка, развитая в [9], [10], работает как для безмассовых [9], так и для массивных [10] материальных полей, она не обладает явной конформной симметрией высших спинов. Таким образом, подход, развитый в этой статье, поднимает важную проблему поиска явно конформной теории, описьвающей взаимодействия высших спинов безмассовой $3 d$-материи и основанной на модулях Фока вместо твистованного присоединенного представления. Можно ожидать, что эта возможность аналогична реализации $2 d$-материальной системы высших спинов [12], первоначально сформулированной в терминах модуля Фока. Также представляется заманчивым предположить, что возникающие в результате модели могут иметь некоторую связь с моделями некоммутативных солитонов [13], [23], обсуждавшимися недавно в контексте некоммутативной фазы теории суперструн [24], которые реализуются в терминах модулей Фока по отношению к некоммутативным пространственно-временным координатам.

Благодарности. М.В.благодарен И. Барсу, М. Гюнайдену и Е. Виттену за полезные обсуждения и центру теоретической физики CIT-USC, где была проделана часть

\footnotetext{
5) Мы не рассматриваем здесь вопросы, касающиеся нормы операторов. Заметим, что оператор (6.7) описывается в $F$ бесконечной матрицей, содержащей конечное число ненулевых элементов, в то время как полиномиальные элементы алгебры звездочного произведения имеют якобиев вид с бесконечным числом ненулевых элементов, но не более чем конечным числом ненулевых диагоналей. Это означает, что полином в алгебре звездочного произведения описывается бесконечной суммой в базисе (6.7). Вопросы о том, насколько хорошо определимы эти бесконечные суммы, иногда оказываются важными.
} 
работы, за гостеприимство. Данное исследование выполнено при частичной поддержке INTAS, грант № 99-1-590, и РФФИ, гранты № 99-02-16207 и № 00-15-96566.

\section{Список литературы}

[1] P.A. M. Dirac. J. Math. Phys. 1963. V. 4. P. 901.

[2] J. Maldacena. Adv. Theor. Math. Phys. 1998. V. 2. P. 231; Int. J. Theor. Phys. 1998. V. 38. P. 1113; hep-th $/ 9711200 ;$ S. Ferrara, C. Fronsdal. Class. Quant. Grav. 1998. V. 15. P. 2153; hep-th/9712239; M. Gunaydin, D. Minic. Nucl. Phys. B. 1998. V. 523. P. 145; hep-th/9802047; S. S. Gubser, I. R. Klebanov, A. M. Polyakov. Phys. Lett. B. 1998. V. 428. P. 105; hep-th/9802109; E. Witten. Adv. Theor. Math. Phys. 1998. V. 2. P. 253; hep-th/9802150.

[3] M. A. Vasiliev. Phys. Lett. B. 1990. V. 243. P. 378; 1992. V. 285. P. 225.

[4] M. A. Vasiliev. High spin gauge theories: start product and AdS space. In: The Many Faces of Superworld. Ed. M. Shifman. Singapore: World Scientific, 1999. P. 533; hep-th/9910096.

[5] M. A. Vasiliev. Int. J. Mod. Phys. D. 1996. V. 5. P. 763.

[6] E.S. Fradkin, V. Ya. Linetsky. Ann. Phys. 1990. V. 198. P. 293.

[7] E.C. Фрадкин, М. А. Васильев. ДАН СССР. 1986. Т. 29. С. 1100; E.S. Fradkin, M. A. Vasiliev. Ann. Phys. 1987. V. 177. P. 63.

[8] M. A. Vasiliev. Fortschr. Phys. 1988. V. 36. P. 33; Nucl. Phys. B. 1988. V. 301. P. 26.

[9] M. A. Vasiliev. Mod. Phys. Lett. A. 1992. V. 7. P. 3689.

[10] S. F. Prokushkin, M. A. Vasiliev. Nucl. Phys. B. 1999. V. 545. P. 385; hep-th/9806236.

[11] E.S. Fradkin, M. A. Vasiliev. Int. J. Mod. Phys. A. 1989. V. 3. P. 2983.

[12] M. A. Vasiliev. Phys. Lett. B. 1995. V. 363. P. 51.

[13] R. Gopakumar, S. Minwalla, A. Strominger. JHEP. 05(2000)020; hep-th/0003160.

[14] M. A. Vasiliev. Class. Quant. Grav. 1994. V. 11. P. 649.

[15] А. В. Барабанщиков, М. А. Васильев, С. Ф. Прокушкин. ТМФ. 1997. Т. 110. № 3. С. 372.

[16] M. A. Vasiliev. Fortschr. Phys. 1987. V. 35. P. 741.

[17] M. Flato, C. Fronsdal. Lett. Math. Phys. 1978. V. 2. P. 421.

[18] M. Gunaydin, D. Minic, M. Zagerman. Nucl. Phys. B. 1999. V. 544. P. 737; hep-th/9810226.

[19] S. E. Konstein, M. A. Vasiliev. Nucl. Phys. B. 1989. V. 312. P. 402.

[20] M. A. Vasiliev. Ann. Phys. (N. Y. ). 1989. V. 190. P. 59.

[21] S. E. Konstein, M. A. Vasiliev. Nucl. Phys. B. 1990. V. 331. P. 475.

[22] S. E. Konstein, M. A. Vasiliev, V. N. Zaikin. JHEP. 12(2000)018; hep-th/0010239.

[23] D. Gross, N. Nekrasov. Solitons in noncommutative gauge theory. hep-th $/ 0010090$.

[24] A. Connes, M. R. Douglas, A. Schwarz. JHEP. 02(1998)003; hep-th/9711162; F. Ardalan, H. Arfaei, M. M. Sheikh-Jabbari. Mixed branes and M(atrix) theory on noncommutative torus. hep-th/9803067; JHEP. 02(1999)016; hep-th/9810072; C-S. Chu, P-M. Ho. Nucl. Phys. B. 1999. V. 550. P. 151; hep-th/9812219; N. Seiberg, E. Witten. JHEP. 09(1999)032; hep-th/9908142. 